\title{
NEUROARQUITECTURA, CREATIVIDAD Y APRENDIZAJE EN EL DISEÑO ARQUITECTÓNICO
}

\author{
NEUROARCHITECTURE, CREATIVITY AND \\ LEARNING IN ARCHITECTONIC DESIGN
}

\section{Laurente Gutiérrez}

\section{Resumen}

Reflexión sobre las nuevas corrientes de la Pedagogía Contemporánea relacionadas a la enseñanza-aprendizaje del Diseño Arquitectónico, desde la Neuroarquitectura. Esta ciencia permitiría a docentes y alumnos conocer y desarrollar sus potencialidades y su relación con la Creatividad, insumo básico de toda producción arquitectónica. Su aplicación racional en el proceso de diseño arquitectónico produciría conocimientos, innovación, originalidad y flexibilidad entre otros aspectos cognitivos; en especial, respecto al espacio interior arquitectónico y a cómo este espacio interior diseñado influye el estado emocional y psicológico en la conducta y/o comportamiento del usuario.

Palabras clave: Neuroarquitectura, creatividad, percepción del espacio; espacio interior arquitectónico, diseño arquitectónico.

\section{Abstract}

Reflection on the new currents of Contemporary Pedagogy related to the teaching-learning of Architectural Design from Neuroarchitecture. This science would allow teachers and students to know and develop their potential and their relationship with Creativity, the basic input of all architectural production. Its rational application in the architectural design process would produce knowledge, innovation, originality and flexibility among other cognitive aspects; especially, regarding the architectural interior space and how this interior space 
designed influences the emotional and psychological state in the behavior and / or behavior of the user.

Key words: Neuroarchitecture; creativity; perception of space; architectural interior space; architectural design.

\author{
"Así pues, el habitar \\ sería en cada caso el fin \\ que preside todo construir" \\ M. HEIDEGger (1994)
}

\section{Introducción}

En principio, la intención de este artículo es pedagógico, pero al mismo tiempo se dirige hacia la búsqueda de la esencia de la enseñanza aprendizaje de la Arquitectura en general y del aprendizaje del Diseño Arquitectónico en particular, en las actuales Escuelas de Arquitectura del país, en su sentido más original y universal. De esta manera se espera encontrar en el análisis de la Neurociencia y de la Psicología y su relación con la Creatividad en el aprendizaje del Diseño Arquitectónico, el sustento epistemológico o el elemento básico del quehacer del diseño en Arquitectura en su etapa fundamental, para planear desde allí la reflexión que debe anteceder a cualquier currículo y que, en última instancia, debe plantearse en cualquier facultad, para permitir a los profesores enmarcar y fundamentar su actuación de enseñanza aprendizaje en el diseño creativo del Espacio y su habitabilidad.

Con esta premisa, se trata de establecer una relación dialógica entre lo pedagógico y lo académico de la en- señanza de la Arquitectura y su relación con las nuevas Teoría del Diseño Arquitectónico, con un enfoque neurocientífico, dirigido específicamente al aprendizaje del Diseño Arquitectónico. El resultado esperado es una formación más humana y científica, para la producción de los espacios habitables en armonía con el bienestar y el confort del ser humano, como protagonista del mismo y su relación dialógica con el entorno y el Medio Ambiente en que se desarrolla.

Para este fin, se siguen las teorias contemporáneas que relacionan el estudio del comportamiento y/o conducta del usuario como reacción fisiopsicológica ante la acción o influencia del espacio arquitectónico, como la Neuroarquitectura en el diseño de espacios habitables. Esta teoría científica nace de la Neurociencia y de la Neuropedagogía, que se ocupan del estudio científico del funcionamiento del cerebro en la etapa de aprendizaje del estudiante; en este caso, del diseño arquitectónico.

Esto conlleva a una reflexión y revisión del diseño curricular de la Carrera de Arquitectura con miras a un cambio en el diseño y contenidos de las competencias, habilidades y actitudes del estudiante y a una integración pedagógica y académica de los cursos básicos y/o complementarios 
que apoyan al Taller de Diseño, como curso formativo estructural.

\section{Figura $N^{\circ} 01$ Esquema Conceptual de la investigación}

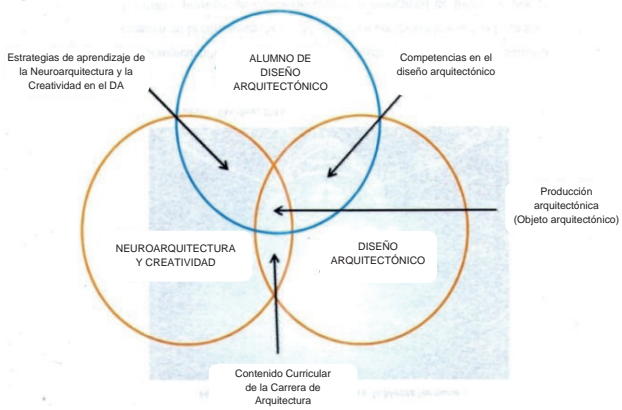

Fuente: Elaboración propia

\section{La Neuroarquitectura y el Diseño Arquitectónico}

Uno de los componentes principales del Diseño Arquitectónico es el diseño del Espacio arquitectónico, cuyo significado etimológico proviene de los vocablos latinos spatium y architectonicus, respectivamente. Es decir, la noción de espacio arquitectónico hace referencia al lugar cuya producción es el objeto de la arquitectura (Pérez y Gardey, 2014). Según los mismos autores, el concepto de espacio arquitectónico se encuentra en permanente revisión por parte de expertos en la materia, lo que origina diversas concepciones.

Al respecto Moreno (2008) dice que hablar del espacio arquitectónico, es hablar del lugar donde habita el hombre y, por lo tanto, se podría definir para este caso, al diseño arquitectónico como la ciencia del diseño del es- pacio habitable o con habitabilidad. Según Landázuri y Maldonado (2004), citando a Castro (1999), habitabilidad es el:

...concepto que se refiere a la satisfacción que uno obtiene en un determinado escenario o grupo de escenarios; es el atributo de los espacios construidos de satisfacer las necesidades objetivas y subjetivas de los individuos y grupos que las ocupan, es decir, las esferas psíquicas y sociales de la existencia estable que podría equipararse a las cualidades medioambientales que permitan el sano desarro1lo físico, biológico, psicológico y social de la persona. (p.90).

Y si de espacio arquitectónico se trata, nos referimos explícitamente en el presente artículo, al espacio interior arquitectónico, que es el que principalmente se diseña en forma académica en las Escuelas de Arquitectura. Por lo general, según Muñoz (2012), los ensayos sobre el espacio arquitectónico inciden tácita y expresamente en el espacio interior como cuestión principal. Y es porque este es primigenio; es lo que necesitamos antes que nada, lo que primero hacemos por la necesidad natural que tenemos de protección, de techo, de interioridad, de privacidad. Así mismo, en ese sentido, el presente artículo no escapa a esa especificidad y es ese el espacio, conceptualizado, diseñado y construido, el que influye directamente en el estado emocional y el comportamiento del usuario que lo consume, que lo recorre y lo usa. 
Al estudio de la acción a través de la percepción del sujeto del espacio percibido y de la reacción de la imagen captada por el cerebro, produciendo sensaciones y comportamientos diversos del sujeto en su conducta, se lo denomina Neuroarquitectura.

\section{El Cerebro Humano y la Percepción del espacio}

Las sensaciones, sean positivas o negativas en el comportamiento humano que le otorga el espacio percibido, según la premisa de Alison Whitelaw (2012), citada por Elizondo y Rivera (2014), se refiere a que: "La calidad del ambiente construido puede afectar el desempeño del cerebro; como la experiencia del ambiente donde se encuentran los personas puede influir en su estado emocional y en su comportamiento".

Las mismas autoras señalan en su investigación, citando a Epstein y Kanwisher (1999), que existe una región del cerebro llamada en inglés Parahippocampal Place Area (PPA). Esta región se encuentra en el hipocampo, la región del cerebro que se dedica a procesar nueva información y almacenar la memoria y los recuerdos. La PPA se activa únicamente con la percepción de lugares, cada vez que un individuo se encuentra en un determinado lugar o recuerda de manera novedosa este espacio. Los autores señalan que la PPA es más activa cuando las personas observan escenas complejas, como paisajes, ciudad, habitaciones con muebles; y estas ex- periencias son almacenadas dentro de nosotros.

\section{Figura $\mathrm{N}^{\circ} 02$ Ubicación del Hipo- campo en el cerebro humano}

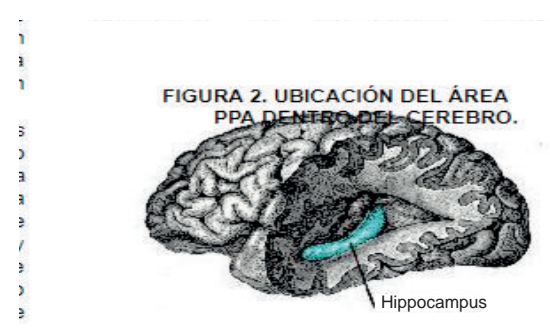

Cuadernos de Arquitectura Año 07 N07 Abril 2017

Fuente: Recuperado de https://commons. wikimedia.org/wiki/File:Gray 739 emphasizing-hippocampus.png

En ese sentido, se puede inferir que la percepción del sujeto del espacio en que participa, es gravitante para generar diversas sensaciones, que dependiendo de la receptividad de su cerebro, hace que éste sea un espacio habitable, que genere confort en sus diversas maneras, que sea adecuado para la función o actividad para lo cual fue destinado, en este caso utilitario, para satisfacer necesidades primarias y/o secundarias del sujeto que percibe. Las características físicas y formales del espacio creado y percibido, dependerá de generar sensaciones positivas y/o negativas en el sujeto observador; de lo cual se desprenderá el diseño creativo experimental del proyectista del referido espacio, como diseñador en una primera fase y como usuario en una 
segunda fase, simultáneamente, durante el proceso creativo.

Según Elizondo y Rivera (2017), en su ponencia sustentada en la ANFA (Academia de Neurociencia de Arquitectura), existen tres factores claves para crear mejores espacios: El primero, es la continuidad del espacio-tiempo. Dentro de nuestro cerebro, específicamente en la región del hipocampo, están ciertas neuronas que reaccionan cuando las personas se encuentran en un lugar o espacio específico; estas neuronas ayudan a fomentar de manera interna una idea o experiencia espacial del mundo exterior a su persona.

El segundo factor clave, es el impacto de la arquitectura en la percepción espacial. Los Arquitectos o diseñadores deben ser sensatos a la hora de diseñar espacios, porque cualquier elemento puede ocasionar algún problema para otro, como en el caso del diseño de espacios para personas con problemas psiquiátricos, en donde algún elemento que les es dificil de identificar puede ocasionar problemas de desubicación, estrés, molestia o nervios.

Como último factor clave, tenemos la iluminación. Desde un aspecto fisiológico, la luz natural es de vital importancia para el cuerpo humano; el no contar con una adecuada iluminación natural puede ocasionar estados de ánimo negativos. Es importante que las personas estén expuestas a 2 mil luxes en un promedio de una hora diaria para neutralizar la depresión que se pue- de ver generada por la falta de iluminación natural.

Finalmente, el surgimiento de la Neuroarquitectura como ciencia, que resulta de unir los estudios de la Neurociencia y de la Arquitectura, ha merecido opiniones diversas sobre la consideración de ella en el diseño de los espacios arquitectónicos y su influencia en el cerebro del sujeto que lo percibe.

Fred Gage (2003) presentó este descubrimiento en una convención de arquitectos, en el Instituto Americano de Arquitectura, enunciando la siguiente idea: "Los cambios en el entorno, cambian el cerebro, por tanto, modifican nuestro comportamiento".

Para Zeisel (2006), el reto actual para la arquitectura es intimar con el cerebro, entender cómo funciona y el por qué hay espacios que favorecen ciertos estados de ánimo. El autor indaga en el campo de la neurociencia para describir el impacto de los edificios y de los espacios en nuestras vidas. Se trata de conocernos por dentro para poder concebir edificios y espacios en consonancia con nuestro bienestar, no sólo físico sino también mental.

Según Barros (2013), el ser humano se ha percatado del influjo que una cierta disposición del espacio tiene sobre la psique, y que, si esta variable se contempla intencionalmente al momento de construir un inmueble, pueden conseguirse resultados específicos. 
Saez (2014), sostiene:

Aunque la neuroarquitectura es un concepto bastante novedoso, que los arquitectos tomen en cuenta principios de salud a la hora de diseñar inmuebles, no lo es. Y es lógico que sea así, porque más del $90 \%$ del tiempo que estamos despiertos al día lo pasamos dentro de edificios, y lamentablemente muchos de los cuales no están pensados y construidos para hacernos sentir bien (p.2).

De las opiniones expuestas, se puede inferir las bondades de la Neuroarquitectura en el Diseño Arquitectónico y la necesidad de contar con ella, para la creación de los espacios habitables. De acuerdo a ello, el diseñador asume un doble rol en el proceso de diseño, como proyectista y como partícipe del espacio a diseñar en forma simultánea durante el acto proyectual; convirtiendo el diseño arquitectónico en una disciplina multidisciplinar. Con la intervención de la Psicología, la Neurología y la propia Arquitectura, se propicia una nueva concepción en la enseñanza y el aprendizaje del Diseño Arquitectónico en las actuales Escuelas de Arquitectura.

\section{La Creatividad y el Diseño Arquitec- tónico. La percepción del espacio construido}

Aún hoy día puede considerarse como muy reciente el inicio de las investigaciones y estudios sobre el campo del conocimiento de la creatividad.
Las primeras aportaciones de autores como De Bono, Osborn o Torrance, datan de la década de los sesenta en adelante, por lo que la aplicación práctica de todo lo hallado a nivel teórico en los centros educativos es todavía escasa e insuficiente.

La creatividad es una preocupación central de la cultura contemporánea, y ella se extiende a muchos campos profesionales. El término creatividad tiene múltiples acepciones y es comprendido de distintas maneras. Ahora se habla de creatividad en las empresas, refiriéndose en este caso a la habilidad para encontrar nuevas rentabilidades en ambientes hostiles, cambiantes y de gran competitividad. En la Biología también se está hablando mucho de creatividad; considerando en ese caso la maravillosa capacidad de los seres vivos de adaptarse para sobrevivir en entornos cambiantes y escasamente predecibles. Se postula aquí que la creatividad sería una condición innata de los seres vivos (Timmling, 2001).

Respecto al diseño arquitectónico como proceso, el mismo Timmling (2009) manifiesta que:

... como proceso representa también una forma de actuar, de intervenir y de transformar el mundo. En dicho proceso se concatenan, simultánea o secuencialmente, procedimientos y operaciones con metas y objetivos y, ellos a su vez, con expectativas y visiones de futuro. Esto incluye valores y motivaciones, siempre en la perspectiva de lograr calidad, confiabi- 
lidad y concordancia en los resultados. Es parte fundamental de este proceso la visualización de las consecuencias y los impactos en los contextos, sean ellos territorial-espaciales, sociales, económicos, culturales o medioambientales, cuyas implicaciones desbordan y muchas veces superan el accionar del propio diseñador. (P.5)

Con respecto a la relación entre creatividad y diseño arquitectónico como procesos, Cubillán (2008), sostiene:

En el proceso de diseño la creatividad juega un papel nuclear porque es expresión de la complejidad del mismo proceso dado que el trabajo es asignado por un cliente o profesor, según sea el caso, pero la persona que interviene debe, desde su propia multidimensionalidad $\mathrm{y}$ complejidad; dar respuesta a la propuesta planteada, tomando en consideración todos los elementos que confluyen en esa obra. La creatividad aquí debe obedecer a un proceso interno de auto organización de elementos que lleven a la coherencia en la expresión, siguiendo los principios, normas y leyes del diseño. En este proceso interno hay que darle un amplio espacio al momento de reflexión, al autoencuentro consigo mismo, a la toma de contacto con su internalidad, al silencio interior, para que afloren las ideas y ocu- pen los espacios de la obra creada. (Pp. 7-8)

En ese sentido, la relación entre Creatividad y Diseño Arquitectónico es simbiótico, inherente e interdependiente; sin creatividad no hay proceso de diseño creativo, innovador y original, y, sin proceso de diseño creativo, no se puede obtener un producto creativo.

Es de interés en esta investigación ocuparnos de la creatividad diseñadora en los Talleres de Arquitectura y, específicamente, en el diseño arquitectónico. Si bien es cierto, y lo dicen muchos estudios al respecto, que el estudio de la creatividad se ha referido a su origen, a su proceso y a su producto; lo que se quiere profundizar es como ésta incide en el proceso del diseño arquitectónico como una herramienta para la investigación en general y para la experimentación en particular, de la disyunción entre sujeto y objeto, lo que Timmling( 2001) denomina la creatividad experimental; es decir, el tratamiento del sujeto creativo y del producto creado como un todo, inseparables a pesar de su diferentes naturaleza, con el propósito de dar soluciones a problemas complejos que emanan de establecer o esclarecer la relación sujeto y objeto.

Si llevamos esta visión filosófica y epistemológica de la creatividad experimental a la relación sujeto-creativo y objeto-espacio en el proceso de diseño arquitectónico, establecemos una relación interdependiente entre el diseñador y el espacio creado, obteniéndose resultados positivos en cuanto 
al uso de espacios más agradables al usuario, generando sensaciones positivas en el uso del espacio percibido que conlleva a una adaptación o adecuación y disfrute del confort, sin llegar a alterar fisiológicamente el estado psicológico y físico del sujeto. Es aquí donde es necesario que el sujeto proyectista se internalice en el proceso de diseño en forma experimental y actúe como sujeto creativo y como sujeto usuario simultáneamente. De lo manifestado, se puede reafirmar que la relación e interdependencia sujetoobjeto se produce en una relación interdisciplinaria entre la Psicología y la Arquitectura; es decir, la Neuroarquitectura, la ciencia que estudia la influencia del objeto-espacio en las sensaciones y conducta de comportamiento humano del sujeto-usuario.

\section{La Psicologia del diseño del espacio (interior) arquitectónico}

El espacio al que nos referimos en este caso, como objeto del proceso de diseño arquitectónico, es el espacio ocupado y usado por el sujeto o usuario; en donde el sujeto, creador y usuario al mismo tiempo durante el proceso creativo y de diseño arquitectónico, conceptualiza el objeto-espacio, pero sin perder de vista que está referido al espacio arquitectónico, en la medida que está contenido en la Arquitectura. Al respecto, Cardoni (2009) dice:

El espacio arquitectónico. Para que podamos comprender la arquitectura desde un punto de vista perceptual tendremos en cuenta aquellas percepciones que se llaman espacio y lugar arquitectónico. Si no está presente esta idea, ya no se considera arquitectura sino escultura, mobiliario, artesanía o diseño industrial. (P.28)

Así mismo, el autor del artículo considera además los dos espacios de la arquitectura: el espacio exterior de la arquitectura, que es ilimitado y en el cual está inserto el objeto construido; en contraposición al espacio interior, que se percibe cerrado, limitado y es una porción del anterior, delimitado por la construcción. La experiencia del espacio interior podrá ser modificada de acuerdo a la posición que elija el observador. En el espacio exterior, la experiencia reviste otra característica, ya que el observador se ubica como al borde desde el cual contempla, más que sentirse rodeado por el espacio.

Entonces, podemos decir que la experiencia del espacio interior estará íntimamente vinculada con el mundo de la percepción y su estructura diferencial (Hesselgren, 1969), entendiéndose como experiencia del espacio interior al resultado de nuestro proceso de percepción e informe de nuestra acción, constituyendo ambos la esencia de la experiencia en el habitar.

Al respecto, Aísa (2012), citando a Pallasmaa, afirma que la experiencia arquitectónica es el "acercarse o enfrentarse a un edificio, más que la percepción formal de una fachada; el acto de entrar y no simplemente del diseño visual de la puerta; mirar al interior o al exterior por una ventana, 
más que la ventana en sí como un objeto material" (p.64). Según este autor, el espacio arquitectónico es un espacio existencial, 'un espacio vivido' y no meramente físico ni estético.

En resumen, el espacio interior de la arquitectura es una experiencia compleja y, como tal, debemos apreciarla más que como una simple percepción. En ella intervienen las diferentes percepciones visuales, hápticas y táctiles, auditivas, kinestésica, y la memoria, como imagen visual que permanece, de lo que tenemos por detrás y no estamos viendo ante nuestros ojos. Por lo tanto, desde el punto de vista del diseño arquitectónico, es espacio interior es aquél en que el diseñador ha de intervenir con el fin de lograr que el habitar del hombre se complete y mejore en calidad, sea cual fuere su uso.

\section{Sensaciones espaciales y su influen- cia en el usuario}

Si hablamos de espacio habitable, es porque el espacio conceptualizado, diseñado y construido, tiene el carácter de "habitar"; un espacio pensado en el hombre (usuario) que reúne las condiciones ideales del confort; para ser más preciso, del confort psicológico, a partir de las características del espacio sociofísico. Es un esquema cognoscitivo de orientación espacial que acepta información del medio sociofisico y dirige acciones predeterminadas mediante las cuales el individuo puede acceder a condiciones psicofisiológicas favorables, sujeto a estadios socioculturales y biológicos, determinado por condiciones contextuales, a juicios de valor (Villalpando-Barceína, 2012).

Al respecto, Aísa (2012) amplía el concepto y el carácter de "habitar" del espacio creado, citando a Heidegger (1951), en su conferencia realizada en Darmstadt (Alemania), titulada: "El hombre y el espacio", en donde hace explícita la necesidad de diálogo entre el construir arquitectónico y el pensar filosófico. Así, el "habitar" es la clave de la unión, al confluir en él tanto el pensar como el construir y, por ende, constituye el proceso de diseño una ciencia multidisciplinar, con la intervención de la Filosofía unida a la Arquitectura.

Al observar el espacio (interior), este nos devuelve configuraciones espaciales de orden sensorial y espacial, que a modo de imágenes reproducidas en nuestro cerebro, influyen en nuestro estado emocional y comportamiento en forma positiva y negativa, según la forma, textura y/o color de los elementos que lo estructuran y se presentan. Esta forma conceptual y filosófica de ver y concebir el espacio interior en el diseño arquitectónico, es poco utilizada por los arquitectos de hoy y muchos menos por el estudiante de arquitectura durante su aprendizaje en las Escuelas de Arquitectura.

\section{Elementos perceptuales como ins- trumentos de diseño del espacio (interior vivencial)}

Robles y Esparza (2014) consideran que la complejidad de la percepción espacial surge a partir del análisis de 
los recursos perceptivos aplicables en el diseño del espacio interior, los cuales serian: los recursos visuales, táctiles, auditivos, olfativos, añadiendo por último el elemento final, la traducción o reconocimiento por medio del efecto memorial, cuyas características se describen sucintamente a continuación:

a. Recursos visuales perceptivos Trasmisible por medio de la vista, y perceptualmente sugerente en el diseño desde los principios básicos, como son: manejo de la forma y el espacio, orden, armonía, limites, escala, secuencia, etc., ligándose directamente a todo tipo de criterios funcionales y estéticos. Es también reconocido y profundamente analizado en el ámbito de la psicología ambiental y social por medio del color y el manejo de la luz, ya sea natural o artificial, y su implementación en los espacios.

b. Recursos táctiles perceptivos La piel, es el órgano más grande de nuestro cuerpo, por lo cual la sensibilidad del ser humano en relación a lo que toca, o simplemente percibe en cuanto al confort del ambiente, constituye una experiencia sensorial muy importante en el diseño de los espacios interiores, ya que hablamos de la capa tangible, por medio de la cual podemos generar ciertos efectos relacionados con las emociones ligadas a la comodidad o al confort. Se trata de elementos como la forma, la densidad y la textura, la solidez, la suavidad, la rugosidad, la temperatura de los materiales; todo lo que es perceptible por medio del tacto.

c. Recursos auditivos perceptivos

Este recurso es aplicable a los espacios como instrumento de diseño acústico, al proveer al espacio de efectos sonoros o, por el contrario, limitarlos o absorberlos, para disminuir su escucha o magnificarlos. Tal es el caso de áreas de trabajo o de concentración, como bibliotecas o, por otra parte, espacios de reproducción y ampliación de sonidos, como salas de cine, teatros, etc.

d. Recursos olfativos perceptivos El olfato es un sentido de percepción global de estímulo compuesto, esto quiere decir que prioriza los olores naturales a los artificiales, y otorga características olfativas de tipo negativas y positivas, de tal forma que lo podemos hacer aplicable en relación a olores y espacio físico. Dentro de estas características positivas y negativas, podemos referir a aquellos aromas que son gratos al olfato y los que generan disgusto. Estas características son completamente aplicables como estrategias dentro del diseño del espacio. Un aroma positivo, podría asociarse a frescura en el ambiente y, por lo tanto, habría una tendencia sobre un olor natural y fresco, vinculado al ambiente y a la cir- 
culación de aire. Un ambiente negativo podría ser aquel que provoca disgusto o malestar $\mathrm{y}$ que se busca evitarlo, como los flujos de viento cruzados.

e. Efecto memorial

La interacción constante entre el espacio, el hombre y, por lo tanto, sus sentidos, otorgan experiencias completas, conexiones sublimes, que Gastón Bachelard identifica como una experiencia del ensueño; es un espacio arquitectónico que puede encuadrar, fortalecer y concentrar todos nuestros pensamientos. Así, la trascendencia de la experiencia perceptiva del espacio conduce a un efecto memorial, al recuerdo, a la evocación de lo ya acontecido, provocando añoranzas, nostalgias, alegrias, etc., es decir, emociones.

A modo de conclusión, todas estas observaciones consideran que el diseño del espacio interior está relacionado con diversas intenciones respecto a los sentidos y su estimulación, potencializando el éxito. Los espacios, en relación a las características de los personas que los experimentarán, requieren del establecimiento de estrategias didácticas y de experimentación que permitan abarcar todos los elementos perceptuales y su repercusión como efecto memorial y psicológico. De esta manera, el espacio en el diseño no se limita al elemento visual, a la belleza del inmueble, a la distinción de las formas por medio de los ojos, sino también a la presencia de elementos más complejos. Generar una propuesta de diseño es contribuir a la generación de experiencias perceptivas, un diseño experiencial que aglutina en una única dimensión no solo espacios compositivamente funcionales y estéticos, sino, también, la construcción de vivencias y sensaciones adecuadas para cada tipo de usuario y ambiente.

\section{Algunas experiencias en el diseño del espacio interior arquitectónico y su influencia en el usuario}

En el 2003, año en el que nació la Academia de la Neurociencia para la arquitectura en San Diego, los expertos en Neurociencia y Arquitectura, establecieron sinergias para entender y conocer cómo el entorno modula el cerebro. De modo que, si los diseños arquitectónicos incorporan principios neurológicos, con ello potenciarán la creatividad y el confort de quienes ocupen esos edificios, en especial sus espacios interiores, donde el usuario disfruta de las sensaciones emotivas que le produce la estructura espacial de los mismos.

Al respecto, la Organización Mundial de la Salud (OMS) establece la existencia de edificios enfermos, calculando en aproximadamente un $30 \%$ los inmuebles que no ayudan a que el organismo mantenga el equilibrio. Cuando eso pasa, es que aparece la enfermedad. Existen numerosas pruebas y estudios que demuestran que la arquitectura puede afectar al conjunto del organismo. De ahí que, desde la OMS, se impulse la construc- 
ción de fincas pensadas para su efectiva función: vivir, trabajar, descansar; para recibir enfermos de Alzhéimer, para educar a los niños, para cuidar a personas convalecientes.

A modo de ilustración, se citan algunos ejemplos sobre la manera en que el diseño de espacios habitables influye en el usuario sensorialmente:
- Se ha visto que los alumnos que estudian en clases con enormes ventanales y mucha luz obtienen mejores resultados que aquellos que lo hacen en aulas más oscuras. Y que los pacientes se recuperan mejor en hospitales diáfanos rodeados de espacios verdes. Una iluminación artificial deficiente no ayuda al cerebro, que debe esforzarse mucho más en la realización de tareas, e influye en una baja productividad y, en las escuelas, en un bajo rendimiento, explica Elisabeth Silvestre, bióloga experta en arquitectura.

- Otro ejemplo, ya en el campo de la medicina, en la Neurología, lo ofrece el neurocientífico Francisco Mora, doctor en Medicina por la Universidad de Granada, quien sostiene que "Todo eso tiene que ver con el funcionamiento del cerebro", y agrega que “... el diseño de espacios puede estimular la creatividad, mantener la atención y concentración de estudiantes y favorecer la relajación".
Elisabeth Silvestre sostiene que "Una iluminación artificial deficiente no ayuda al cerebro que debe esforzarse mucho más; eso en las empresas puede influir en una baja productividad y en las escuelas en un bajo rendimiento".

- La altura del techo, de los espacios habitables en general, influyen en la conducta, comportamiento y pensamiento del usuario. Los espacios con techos altos en relación a su superficie, facilitan el desarrollo de la creatividad y. por lo tanto. la abstracción en actividades artísticas y de diseño; mientras que, los espacios con techos bajos, facilitan la concentración y el análisis. En ese sentido, por ejemplo, los primeros son adecuados para Talleres de Arte o Talleres de Diseño de las Escuelas de Arquitectura y, los segundos, para Salas de Operaciones o Quirófanos, de locales de Salud.

Sutil y Perán (2012, p.7), señalan que una iluminación adecuada de los espacios arquitectónicos es claramente necesaria para el correcto desarrollo de los usos a los que están destinados, aunque existe la creencia de que la luz fluorescente (que es similar a la luz natural espectralmente) conduce a mayor nivel de productividad y tiene mejor impacto. Así mismo, estos autores agregan que el color es una 
de las dimensiones más económicas y más fácilmente manipulables en el diseño del espacio arquitectónico. En líneas generales, se puede establecer que las habitaciones con colores claros son percibidas como más espaciosas y transmiten menor percepción de masificación que idénticos espacios pintados con colores oscuros.

- Finalmente, como caso muy singular, que amerita ser mencionado, respecto a la influencia del ruido y la iluminación en el interior de los espacios, se tienen la implementación de equipos de control de sonidos e iluminación para su mitigación en salas UCI (Unidad de Cuidados Intensivos) y de recién nacidos, comprobándose que aquellos elementos tienen un efecto positivo sobre el desarrollo de los bebés. Así, hoy muchas salas para prematuros en locales de atención a la salud, se desarrollan teniendo en cuenta estos criterios.

\section{E1 Aprendizaje del Diseño Arqui- tectónico en las Escuelas de Ar- quitectura. Un enfoque Neuro-psi- cológico arquitectónico}

\section{La influencia de la Arquitectura Posmoderna en la enseñanza de la arquitectura}

El aprendizaje de Diseño Arquitectónico en las Escuelas de Arquitectura de América Latina y del país, para ser más específico, en estos últimos años ha sido influenciado por la denominada Arquitectura Posmoderna, que nace en 1970 y se consolida a partir del año 1980. Para Jencks (1986), uno de los arquitectos más representativo de esta corriente, la arquitectura posmoderna entre otros aspectos; es aquella caracterizada formalmente por la similitud con las envolventes y plasticidades curvas y el uso intensivo de las metáforas, para expresar el significado del objeto diseñado y edificado contextualmente. Es decir, la mayor preocupación de la forma del objeto arquitectónico, y la menor preocupación en el tratamiento del espacio interior, que a la larga resultaba menos elaborada en función de las necesidades del usuario a satisfacer.

Sobre el tema, agrega Padilla (2015), citando a Jencks (1984), “... el posmodernismo en la arquitectura y el diseño urbano tiende a estar descaradamente orientado hacia el mercado porque ese es el lenguaje primordial de comunicación de nuestra sociedad" (citado por Harvey, 2012, p.96). La envolvente exterior se vuelve el elemento principal de los edificios, la fachada es protagonista. El problema que se generó fue no saber diferenciar la delgada línea entre lo necesario y lo superfluo. No existía una "honestidad" arquitectónica, los edificios no revelaban su función, el interior y el exterior podrian ser totalmente diferentes, no importaba que se mantuviera una coherencia ni mucho 
menos una comunicación entre entorno/edificio.

La misma autora agrega en su investigación al respecto, que no es prioridad en el proceso de diseño ...las necesidades del usuario, las cuales frecuentemente son relegadas a segundo término, pasando de una arquitectura funcional (moderna) a otra puramente visual (posmoderna). Reorientar los enfoques y señalar la importancia de considerar el bienestar del usuario en la arquitectura mejora la calidad de vida y el disfrute de los espacios. (P. 2).

Finalmente, concluye que la idea central es demostrar que la arquitectura cuenta con alternativas y no solo es cuestión de moda, como la establecida durante las últimas décadas, basada en una arquitectura que ha sido bandera de imagen, espectáculo y artificio formal. Pero la autora no solamente constata una realidad, sino apela a un cambio de rumbo sustentada en convicciones ético-morales. Estas parten de considerar que la preocupación primordial del arquitecto es el usuario, principio desatendido en los últimos tiempos. La cuestión es, por tanto, reemplazar la imagen espectacular por la calidad de vida como objetivo prioritario de las nuevas generaciones de arquitectos, a partir de una nueva metodología del diseño.

\section{E1 diseño del espacio (interior) arquitectónico: Un problema de sensibilidad y percepción en el es- tudiante de arquitectura}

Teniendo en cuenta lo expuesto, actualmente la metodología del diseño arquitectónico empleados en los Talleres de Arquitectura, en general, pretende que en el aprendizaje del estudiante se planteen soluciones arquitectónicas con un fuerte énfasis en la percepción y el diseño exterior del objeto arquitectónico, pasando a segundo plano la percepción y el diseño del espacio interior del mismo. El resultado es un producto arquitectónico con una suerte de manejo composicional exterior del mismo, que responde a una imagen determinada previamente, usando estrategias y/o técnicas de presentación formal y espacial, en base a códigos, símbolos, metáforas y/o analogías. Es decir, es un producto arquitectónico como solución, para ser visto desde afuera; en otras palabras: un diseño hecho desde la vista y para ser visto.

Según Ayora y Urzais (1997, p.87), según el método de diseño adoptado, los estudiantes de arquitectura son entrenados para crear espacios; por lo tanto, deben acostumbrarse desde un principio a trabajar con las tres dimensiones que lo definen. Además, parece lógico establecer que si vemos, percibimos y pensamos de manera tridimensional, naturalmente deberíamos proyectar de la misma manera, manejando los 
conceptos propios de la composición arquitectónica (espacio, volumen, plano, linea, punto, etc.), siempre de modo integral y desde la perspectiva real en tres dimensiones.

La falta de estrategias y/ o técnicas de percepción y sensibilidad en el estudiante de Arquitectura, específicamente, entre otros aspectos, en la conceptualización del espacio interior y en su materialización física-espacial, da como resultado que logren soluciones arquitectónicas de escasa creatividad formal y estructural, lo que muchas veces resulta impactando negativamente en la sensibilidad y confort del usuario, para realizar las funciones y/o actividades a la que supuestamente estuvo diseñado. La ausencia de herramientas cognitivas y actitudinales en el aprendizaje, genera la formación de un estudiante carente de sensibilidad y percepción en su formación en el diseño del espacio interior.

El modelo tradicional del Taller de Arquitectura está convirtiéndose actualmente en un espacio de aprendizaje interdisciplinar, donde los estudiantes se forman colaborando con otros que proceden de diferentes disciplinas. El principal propósito de este tipo de talleres consiste en establecer conexiones entre los estudiantes de diferentes cursos y otras carreras para potenciar la colaboración mutua a partir del desarrollo de un proyecto; proyectos de alta complejidad funcional y especialización (Hospitales Especializados, Casas de Reposo, Institutos Penitenciarios, Instituciones de educación, etc.) que requieren la presencia, aparte de los de arquitectura, de alumnos de otras especialidades comprometidas con esta última, como son la psicología, la medicina, la psiquiatría, la pedagogía, etc.; todo lo cual implementaría la formación integral del estudiante en el diseño de complejos arquitectónicos de mediana y alta complejidad funcional.

Según se puede apreciar en la figura que se indica, de una investigación realizada (Gutiérrez, 2016) en los Talleres de Diseño Arquitectónico FAU-URP, en relación al uso de estrategias y/o técnicas para el desarrollo de la creatividad en el proceso de diseño arquitectónico, se pudo advertir el uso intensivo de la maqueta como técnica tridimensional para la conceptualización y materialización física-espacial del espacio interior en los primeros ciclos (I-II-II y IV), un uso de mediana intensidad en los ciclos intermedios (V y VI) y una muy baja en los ciclos restantes, limitándose principalmente a la representación formal de la envolvente y los espacios exteriores(urbanos). 


\section{Figura $\mathrm{N}^{\circ} 03$ Correlación de dimensiones de la Creatividad en el Proceso de Diseño Arquitectónico}

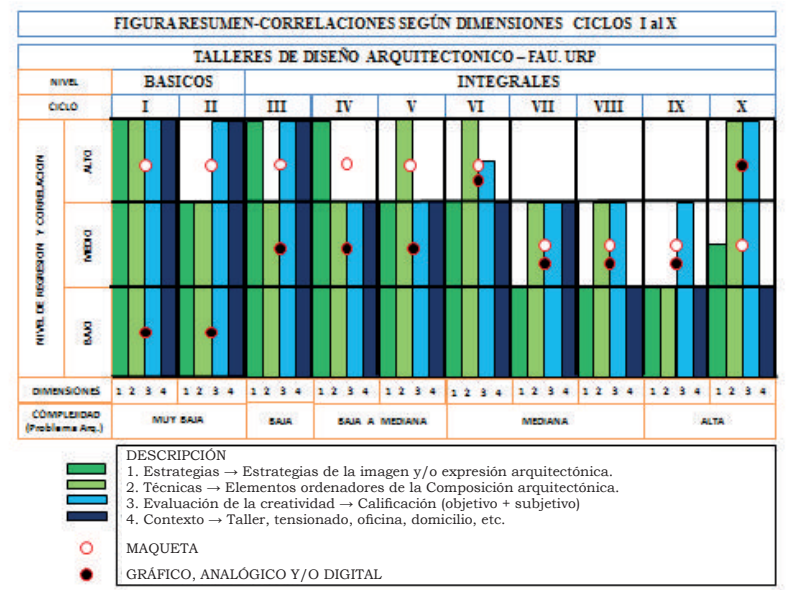

Fuente: Gutiérrez (2016). "Estrategias y Técnicas para el Desarrollo de la Creatividad en el Proceso de Diseño Arquitectónico en los Talleres de Diseño Arquitectónico FAU-URP (Tesis doctoral). Universidad Nacional Federico Villarreal, Lima Perú.

A esto se agrega la falta en los estudiantes de arquitectura, del manejo como medio procedimental, la expresión gráfica de tres dimensiones desde el inicio en la búsqueda creativa de la solución más adecuada mediante el boceto y/o el apunte. Ello, sumado al insuficiente entrenamiento en la formación de conceptos espaciales, y al abuso de las metodologías del diseño empleados en el Taller que no les permite lograr soluciones alternativa al diseño de espacios interiores apropiados. Este último aspecto constituye un elemento primordial en el desarrollo de la sensibilidad y creatividad de los alumnos, pero que no se desarrolla de manera constante en la formación de la Carrera, principalmente en los primeros ciclos o años; razón por la cual el estudiante no tiene la capacidad de interiorizar, y menos de manera creativa, el diseño de espacios interiores para los problemas arquitectónicos muchos más complejos en los últimos años de su formación.

\section{Algunas consideraciones y reco- mendaciones finales}

El aprendizaje del diseño arquitectónico, desde el punto de vista pedagógico, no es ajeno a la revolución de cambio y avance de la ciencia y la tecnología. Por el contrario, las escuelas de arquitectura se han visto comprometidas a actualizar los método de enseñanza-aprendizaje de la Arquitectura en general y del aprendizaje del diseño arquitectónico en 
particular; lo cual se refleja en los cursos afines y/o complementarios señalados en la estructura curricular, en los contenidos y en los métodos de aprendizaje de los mismos.

La ausencia de disciplinas afines al diseño arquitectónico que pudieran reforzar el aprendizaje del mismo; esencialmente en el diseño del espacio interior habitable, como la implementación de la neurociencia y la psicología como estrategias y/o técnicas en la concepción espacial del mismo, ha producido limitaciones en el estudiante para encarar con precisión y creatividad el diseño adecuado y confortable para el sujeto-usuario específico; es decir, el diseño del espacio interior identificado y relacionado con la sensibilidad, comportamiento y conducta del mismo como insumos de diseño. En ese sentido, la enseñanza y el aprendizaje del diseño arquitectónico actual se ha diversificado, y no se puede obviar disciplina afines con la arquitectura y menos con el diseño de espacios (interior) para el hombre, con lo cual es menester estudiar y proponer cursos complementarios al diseño arquitectónico por el carácter multidisciplinario de la Arquitectura actual.

En ese sentido, se estaría apostando por un Taller de Diseño Arquitectónico multidisciplinario, con la participación de alumnos de otras especialidades y/o carreras, promoviendo las competencias transversales asociadas al trabajo en equipos interdisciplinares que complementen las especificidades de cada titulación, y por un taller de diseño arquitectónico con un espacio de aprendizaje interdependiente, donde diferentes tipos de participantes (alumnos) e instituciones pueden trabajar conjuntamente de forma presencial y/o a distancia en el desarrollo de proyectos de alta complejidad y envergadura.

Por lo tanto, un reajuste $\mathrm{y} / \mathrm{o}$ actualización de los cursos de diseño arquitectónico conlleva a una revisión de los mismos y de los cursos afines a los talleres de diseño en particular y de los currículos en general, a fin de darles en su contenido y desarrollo un enfoque neuropsicológico y arquitectónico. En este caso, con la finalidad de formar estudiantes con sensibilidad, analíticos y creativos como proyectistas y partícipes (usuarios) en la concepción del espacio interior, con sus sensaciones, conductas y comportamientos respectivos. 


\section{REFERENCIAS BIBLIOGRÁFICAS}

Aisa, I (2012). "Arquitectura y Sensibilidad. Filosofia en la arquitectura de Juhani Pallasmaa”, En: Revista de Filosofia. N 45. 2012. [13] Universidad de Sevilla.

Ayora, S y Urzaiz, E (1997). Enseñanza-aprendizaje del diseño arquitectónico. En: revista Educación y Ciencia (Enero-Junio 1997) de la Facultad de Educación de la Universidad de Yucatán, México

Cardoni, Z. I. (1 de Agosto de 2009). "El espacio vivencial del hombre entre la psicología y la arquitectura". De Sitio Web de la Universidad Argentina John F. Kennedy: Escuela de Psicología. Recuperado de:

http: / / www2.kennedy.edu.ar/departamentos/psicoanalisis / tfi /TFI_ Cardoni.pdf

Elizondo, A. y Rivera, N. (2017) “El Espacio Físico y la Mente: Reflexión sobre la Neuroarquitectura". Ponencia presentada y publicada por Cuadernos de Arquitectura y Asuntos Urbanos, Revista de la Facultad de Arquitectura, Universidad Autónoma de Nuevo León, Año 07, Núm. N Abril 2017, Pág 41.

Gage, F. (2003). "Neurociencia y Arquitectura", Ponencia presentada en la Convención Nacional de Arquitectos, Instituto Americano de Arquitectura. San Diego, California, USA

Gonzales, L. (2008). "El proceso creativo en el diseño arquitectónico", en: Facultad de Arquitectura, revista de la Universidad del Zulia, Venezuela

Gutiérrez, L. (2016). “Estrategias y Técnicas para el Desarrollo de la Creatividad en el Proceso de Diseño Arquitectónico en los Talleres de Diseño Arquitectónico-FAU-URP”, tesis Doctoral. Lima: Universidad Nacional Federico Villarreal.

Jenks, Ch. (1986). El lenguaje de la arquitectura posmoderna. Barcelona: Gustavo Gili.

Moreno, S. (2008). "La habitabilidad urbana como condición de calidad de vida”, en: Palapa, vol. III, núm. II, julio-diciembre, 2008, pp. 47-54 Universidad de Colima México.

Oppenheimer. A. (2013). Basta de historias! La obsesión Latinoamericana con el pasado y las 12 claves del futuro. $1^{\text {a }}$ ed. Buenos Aires: Debate, 2013

Padilla, Karina (2015). "Más allá de lo icónico: las necesidades del usuario frente a la arquitectura espectáculo a través de la vivienda de interés social”. Tesis de Maestría, Universidad Autónoma de Nuevo León, México 
Robles, L. y Esparza, E. (2015). "Experiencia perceptiva en el diseño de los espacios interiores”, en: Revista: Interiorgráfica de la división de Arquitectura, Arte y Diseño de la Universidad de Guanajuato, Año 16, Nº16, Enero - diciembre 2015

Sutil, L. y Perán, J. (2012). Neuroarquitectura y comportamiento del consumidor: Una propuesta de modelo de diseño, Universidad Rey Juan Carlos Barcelona, 2012

Villapando, A y Barceína, E (2012), “Teoría del Diseño II Psicología y Arquitectura. Notas Breves: Confort Psicológico”. México: Posgrado de Arquitectura, Universidad Nacional Autónoma de México. 